\title{
Microwave-induced magnetooscillations and absolute negative conductivity in the multisubband two-dimensional electron system on liquid helium
}

\author{
Yu.P. Monarkha ${ }^{1}$ \\ ${ }^{1}$ Institute for Low Temperature Physics and Engineering, $4^{7}$ Lenin Avenue, 61103 Kharkov, Ukraine
}

\begin{abstract}
It is shown that a nonequilibrium filling of an upper surface subband induced by the microwave resonance can be the origin of the absolute negative conductivity and zero-resistance states for the two-dimensional electron system on liquid helium under magnetic field applied normally. Contrary to the similar effect reported for semiconductor systems, an oscillating sign-changing correction to the dc-magnetoconductivity appears due to quasi-elastic iter-subband scattering which does not involve photons. The analysis given explains remarkable magnetooscillations and zero-resistance states recently observed for electrons on liquid helium.
\end{abstract}

PACS numbers: 73.40.-c,73.20.-r,73.25.+i, 78.70.Gq

Magnetooscillations and absolute negative conductivity induced by microwave (MW) radiation under magnetic field applied perpendicular to a thin semiconductor film were predicted theoretically by Ryzhii already in 1969플. For a long while, these effects were not observed experimentally. Quite recently, magnetooscillations and the effect of vanishing resistivity (zero-resistance states) induced by MW radiation were observed in semiconductor two-dimensional (2D) electron systems of ultrahigh mobility ${ }^{2}$. It was shown that the negative conductivity $\left(\sigma_{x x}<0\right)$ by itself suffices to explain the zero-dcresistance state (ZRS) observed ${ }^{3}$. There are also alternative explanations which refer to an excitonic mechanism for electron pairing ${ }^{2}$.

Recently, microwave-induced magnetooscillations ${ }^{4}$ and even vanishing resistivity $\underline{4.5}$ were observed in the quasi2D electron system on liquid helium. Despite striking similarity of these results to those obtained for semiconductor systems, there are important differences in experimental conditions. First, it should be emphasized that surface electrons (SEs) on helium is a nondegenerate system with strong Coulomb interaction. Therefore, it is doubtful whether electron paring can be realized in such a system. The second important difference is that for semiconductor $2 \mathrm{D}$ systems magnetooscillations and ZRS were predicted and observed for a quite arbitrary MW frequency $\omega$ without excitation of higher subbands, while for SEs on liquid helium the effect is observed only when the MW frequency is under the resonance condition for exciting electrons to the second surface subband: $\hbar \omega=\Delta_{2}-\Delta_{1}$ (here $\Delta_{l}$ describes energy spectrum of surface levels, and $l=1,2, \ldots)$. This means that the theory of photon-stimulated impurity scattering $\frac{1}{\underline{1}}$ which leads to magnetooscillations and negative conductivity in semiconductor 2D systems is not sufficient for explaining ZRS of SEs on liquid helium, at least under conditions of the experiment.

In this Letter we show that additional electron population of the first excited subband induced by the MW resonance provides a possibility for ordinary impurity iter-subband scattering to result in an oscillating signchanging correction to $\sigma_{x x}$. At high enough MW powers and at certain magnetic fields, this correction leads to absolute negative conductivity and, according to Ref $\frac{3}{2}$, to the zero-resistance state observed for SEs on liquid helium 4,5 .

In order to understand the physics of appearing of the sign-changing correction to the dc-magnetoconductivity under the MW resonance condition, it is instructive to remind the explanation given for photon-induced impurity scattering $\stackrel{\underline{1}}{-}$. Consider a multisubband $2 \mathrm{D}$ electron system under perpendicular magnetic field $\mathbf{B}$, and assume that its energy spectrum is described by $\varepsilon_{l, n}=$ $\Delta_{l}+\hbar \omega_{c}(n+1 / 2)$, where $\omega_{c}$ is the cyclotron frequency $e B / m_{e} c$ and $n=0,1,2, \ldots$. For photon-induced impurity scattering within the ground subband, electron current $j_{x}$ is proportional to $q_{y} \delta\left[\hbar \omega_{c}\left(n-n^{\prime}\right)+\hbar \omega+e E l_{B}^{2} q_{y}\right]^{\frac{1}{}}$, where $\hbar \mathbf{q}$ is the momentum exchange, $l_{B}^{2}=\hbar c / e B, E$ is the in-plane driving electric field, $\hbar \omega$ is the photon energy. The delta-function represents the energy conservation which takes into account Landau level tilting in the driving field; $e E l_{B}^{2} q_{y} \equiv e E\left(X-X^{\prime}\right) \equiv q_{y} v_{H}$ (here $\left.v_{H}=c E / B\right)$. Depending on the sign of $\hbar \omega_{c}\left(n-n^{\prime}\right)+\hbar \omega$, the $q_{y}$ could be positive or negative which was the explanation for changing the direction of the current.

Under the MW resonance condition $\hbar \omega=\Delta_{2}-$ $\Delta_{1}$, a nonequilibrium filling of the first excited subband gives rise to usual inter-subband impurity scattering which is restricted by a similar delta-function $\delta\left[\hbar \omega_{c}\left(n-n^{\prime}\right)+\Delta_{2,1}+e E l_{B}^{2} q_{y}\right]$, where $\Delta_{l, l^{\prime}}=\Delta_{l}-\Delta_{l^{\prime}}$. Therefore, the sign of $q_{y}$ depends on $\hbar \omega_{c}\left(n-n^{\prime}\right)+\Delta_{2,1}$, and, if for ordinary impurity scattering there is a current correction linear in $q_{y}$, it will be an oscillating signchanging correction. We shall show that a similar correction to electron conductivity appears when an additional filling of the second surface subband exceeds that given by the Boltzmann factor: $N_{2}-N_{1} e^{-\Delta_{2,1} / T_{e}}>0$. In this case, the MW affects electron transport in an indirect way, just changing subband occupation numbers $N_{l}$.

SEs on liquid helium are scattered quasi-elastically by helium vapor atoms and by capillary wave quanta (ripplons). In this work we consider only scattering at vapor atoms which is similar to usual impurity scattering in semiconductor systems. It is conventionally described by 
the effective potential $V\left(\mathbf{R}_{e}-\mathbf{R}_{a}\right)=V_{a} \delta\left(\mathbf{R}_{e}-\mathbf{R}_{a}\right)$ and by the interaction Hamiltonian

$$
V_{\mathrm{int}}^{(a)}=V_{a} \sum_{\mathbf{K}, \mathbf{K}^{\prime}} e^{i \mathbf{K} \mathbf{R}_{e}} a_{\mathbf{K}^{\prime}-\mathbf{K}^{\dagger}}^{\dagger} a_{\mathbf{K}^{\prime}},
$$

where $\hbar \mathbf{K} \equiv\left\{\hbar K_{z}, \hbar \mathbf{q}\right\}$ is the $3 \mathrm{D}$ momentum of a vapor atom, and $a_{\mathbf{K}}^{\dagger}\left(a_{\mathbf{K}}\right)$ is the creation (destruction) operator of vapor atoms. The $2 \mathrm{D}$ vector $\hbar \mathbf{q}$ represents momentum exchange at a collision.

Considering a multisubband 2D system under the MW resonance condition, we cannot use the conventional linear-response theory. As we shall see, even the effective electron temperature approximation will not help to obtain a sign-changing correction to $\sigma_{x x}$. For arbitrary population of surface subbands $n_{l}=N_{l} / N_{e}$, it is convenient to use the force-balance method (for details, see Ref $\left.{ }^{6}\right)$. For an isotropic system, this method assumes that the average kinetic friction $\mathbf{F}_{\text {scat }}$ acting on electrons is proportional to the electron current, which can be written as $\mathbf{F}_{\text {scat }}=-N_{e} m_{e} \nu_{\text {eff }} \mathbf{v}$ av , where $\mathbf{v}_{\text {av }}$ is the average electron velocity, $N_{e}$ is the total electron number, $m_{e}$ is the electron mass. The proportionality factor $\nu_{\text {eff }}(B)$ represents the effective collision frequency which generally depends on magnetic field $B$ (quantum limit) and even on the areal electron density $n_{s}$ when many-electron effects are considered. Once $\nu_{\text {eff }}(B)$ is found, the conductivity tensor can be obtained from equations similar to the conventional Drude equations.

For noninteracting electrons, $\mathbf{F}_{\text {scat }}$ can be found by evaluating the momentum gained by scatterers. Consider an arbitrary electron distribution $f_{l}(\varepsilon)=N_{l} f(\varepsilon)$, where $\varepsilon$ is the in-plane energy. We shall use the condition $\int f_{l}(\varepsilon) D_{l}(\varepsilon) d \varepsilon=N_{l}$, where $D_{l}(\varepsilon)$ is the densityof-state function $D_{l}(\varepsilon)=-\left(2 \pi^{2} l_{B}^{2} \hbar\right)^{-1} \sum_{n} \operatorname{Im} G_{l, n}(\varepsilon)$, and $G_{l, n}(\varepsilon)$ is the single-electron Green's function for the corresponding subband. In the Born approximation, we obtain

$$
\mathbf{F}_{\mathrm{scatt}}=\frac{\hbar^{2} \nu_{0}^{(a)}}{m_{e}} N_{e} \sum_{l, l^{\prime}} n_{l} s_{l, l^{\prime}} \sum_{\mathbf{q}} \mathbf{q} S_{l, l^{\prime}}\left(\mathbf{q}, \omega_{l, l^{\prime}}\right),
$$

where $n_{l}=N_{l} / N_{e}, s_{l, l^{\prime}}$ represents $\sum_{K_{z}}\left|\left(e^{i K_{z} z}\right)_{l, l^{\prime}}\right|^{2}$ normalized according to Ref. $\underline{\underline{7}}$,

$$
\begin{gathered}
S_{l, l^{\prime}}(\mathbf{q}, \omega)=\frac{1}{\pi^{2} l_{B}^{2} \hbar} \int d \varepsilon f(\varepsilon) \sum_{n, n^{\prime}} J_{n, n^{\prime}}^{2}\left(x_{q}\right) \times \\
\times \operatorname{Im} G_{l, n}(\varepsilon) \operatorname{Im} G_{l^{\prime}, n^{\prime}}(\varepsilon+\hbar \omega), \\
J_{n, n+m}^{2}(x)=\frac{n !}{(n+m) !} x^{m} e^{-x}\left[L_{n}^{m}(x)\right]^{2},
\end{gathered}
$$

$L_{n}^{m}(x)$ is the associated Laguerre polynomials, $x_{q}=$ $q^{2} l_{B}^{2} / 2$, and $\nu_{0}^{(a)}$ is the collision frequency in the absence of the magnetic field. If the collision broadening of Landau levels does not depend on $l$, the $S_{l, l^{\prime}}(\mathbf{q}, \omega)$ coincides with the dynamical structure factor $S(\mathbf{q}, \omega)$ of a nondegenerate $2 \mathrm{D}$ system of noninteracting electrons.

For SEs on liquid helium, electron-electron collision frequency is much higher than $\nu_{\text {eff }}$. In this case, $S_{l, l^{\prime}}(\mathbf{q}, \omega)$ coincides with its equilibrium form $S_{l, l^{\prime}}^{(0)}(q, \omega)$ and $f(\varepsilon)$ equals to $A e^{-\varepsilon / T_{e}}$ only in the center-of-mass reference frame moving with the drift velocity $\mathbf{v}_{\text {av }}$ (here $A$ is a normalization factor). Because of the Galilean invariance along the interface, in the laboratory frame, $S_{l, l^{\prime}}(\mathbf{q}, \omega)$ (as well as the dynamical structure factor ${ }^{6}$ ) acquires the Doppler shift: $S_{l, l^{\prime}}(\mathbf{q}, \omega)=S^{(0)}\left(q, \omega-\mathbf{q} \cdot \mathbf{v}_{\mathrm{av}}\right)$. The $\hbar \mathbf{q} \cdot \mathbf{v}_{\mathrm{av}}$ represents an additional energy exchange at a collision which appears in the center-of-mass reference frame.

In the cumulant approach ${ }^{8}, \operatorname{Im} G_{l, n}(\varepsilon)$ has a Gaussian shape with the Landau level broadening $\Gamma_{l}$ independent of $n$ (for the lowest surface subband, $\Gamma_{1}=\sqrt{\frac{2}{\pi} \hbar^{2} \omega_{c} \nu_{0}^{(a)}}$ ). In this case,

$S_{l, l^{\prime}}^{(0)}(q, \omega)=\frac{2 \pi^{1 / 2} \hbar}{Z_{\|}} \sum_{n, n^{\prime}} \frac{J_{n, n^{\prime}}^{2}\left(x_{q}\right)}{\Gamma_{l, l^{\prime}}} e^{-n \hbar \omega_{c} / T_{e}} I_{l, l^{\prime} ; n^{\prime}-n}(\omega)$,

where $Z_{\|}^{-1}=1-e^{-\hbar \omega_{c} / T_{e}}$,

$$
I_{l, l^{\prime} ; m}(\omega)=\exp \left[-\left(\frac{\hbar \omega-m \hbar \omega_{c}-\Gamma_{l}^{2} / 4 T_{e}}{\Gamma_{l, l^{\prime}}}\right)^{2}+\frac{\Gamma_{l}^{2}}{8 T_{e}^{2}}\right],
$$

and $\Gamma_{l, l^{\prime}}^{2}=\left(\Gamma_{l}^{2}+\Gamma_{l^{\prime}}^{2}\right) / 2$. We assume that $\Gamma_{l} \ll T$.

Using $S_{l, l^{\prime}}^{(0)}(q,-\omega)=e^{-\hbar \omega / T_{e}} S_{l^{\prime}, l}^{(0)}(q, \omega)$, Eq. (2) can be represented as

$$
\begin{gathered}
\mathbf{F}_{\text {scatt }}=\frac{\hbar^{2} \nu_{0}^{(a)}}{2 m_{e}} N_{e} \sum_{l, l^{\prime}} s_{l, l^{\prime}} \sum_{\mathbf{q}} \mathbf{q} S_{l, l^{\prime}}^{(0)}\left(q, \omega_{l, l^{\prime}}-\mathbf{q} \cdot \mathbf{v}_{\mathrm{av}}\right) \times \\
\times\left[n_{l}-n_{l^{\prime}} e^{-\Delta_{l, l^{\prime}} / T_{e}} e^{\hbar \mathbf{q} \cdot \mathbf{v}_{\mathrm{av}} / T_{e}}\right] .
\end{gathered}
$$

For slow drift velocities, $\hbar \mathbf{q} \cdot \mathbf{v}_{\mathrm{av}} \ll \Gamma_{l, l^{\prime}}$ and $\hbar \mathbf{q} \cdot \mathbf{v}_{\mathrm{av}} \ll$ $T_{e}$, linear in $\mathbf{v}_{\text {av }}$ terms of $\mathbf{F}_{\text {scatt }}$ and the effective collision frequency $\nu_{\text {eff }}$ can be easily obtained from Eq. (4). The linear term obtained expanding $e^{\hbar \mathbf{q} \cdot \mathbf{v}_{\mathrm{av}} / T_{e}}$ represents the usual result of which the effective collision frequency is always positive.

There is another term originated from the expansion of $\left(n_{l}-n_{l^{\prime}} e^{-\Delta_{l, l^{\prime}} / T_{e}}\right) \mathbf{q} S_{l, l^{\prime}}^{(0)}\left(q, \omega_{l, l^{\prime}}-\mathbf{q} \cdot \mathbf{v}_{\mathrm{av}}\right)$ in $\hbar \mathbf{q} \cdot \mathbf{v}_{\mathrm{av}} / \Gamma_{l, l^{\prime}}$. This is the linear in $\mathbf{q}$ term we were searching for. It is similar to the corresponding term of Ref. $\frac{1}{m}$ obtained for photon-induced impurity scattering because $S_{l, l^{\prime}}^{(0)}\left(q, \omega_{l, l^{\prime}}-\mathbf{q} \cdot \mathbf{v}_{\text {av }}\right)$ initially contained the $\delta$ function representing energy conservation. Expanding $I_{l, l^{\prime} ; m}\left(\omega_{l, l^{\prime}}-\mathbf{q} \cdot \mathbf{v}_{\mathrm{av}}\right)$ of Eq. (4), we obtain the factor $\Delta_{l, l^{\prime}}-\left(n^{\prime}-n\right) \hbar \omega_{c}-\Gamma_{l}^{2} / 4 T_{e}$ which has different signs at the opposite sides of the point where it equals zero. 
If heating of the electron system is small $\left(T_{e} \ll \Delta_{2,1}\right)$, we can use the two-subband model $(l=1,2)$ and find the sign-changing correction to $\nu_{\text {eff }}$

$$
\begin{aligned}
\delta \nu_{\mathrm{eff}}= & -\frac{\nu_{0}^{(a)} \hbar^{2} \omega_{c}^{2}}{\sqrt{\pi}} \frac{s_{1,2}}{\Gamma_{2,1}^{2}}\left(n_{2}-n_{1} e^{-\Delta_{2,1} / T_{e}}\right) \times \\
& \times\left[\operatorname{coth}\left(\frac{\hbar \omega_{c}}{2 T_{e}}\right) \Phi\left(\omega_{c}\right)+\Theta\left(\omega_{c}\right)\right],
\end{aligned}
$$

where

$$
\begin{aligned}
& \Phi\left(\omega_{c}\right)=\delta F_{2,1}\left(\omega_{c}\right)-e^{\Delta_{2,1} / T_{e}} \delta F_{1,2}\left(\omega_{c}\right) \\
& \Theta\left(\omega_{c}\right)=\delta H_{2,1}\left(\omega_{c}\right)-e^{\Delta_{2,1} / T_{e}} \delta H_{1,2}\left(\omega_{c}\right) .
\end{aligned}
$$

For $\Delta_{l, l^{\prime}}>0$,

$$
\delta F_{l, l^{\prime}}\left(\omega_{c}\right)=\sum_{m=0}^{\infty} I_{l, l^{\prime} ; m}\left(\Delta_{l, l^{\prime}}\right) \frac{\left(\Delta_{l, l^{\prime}}-m \hbar \omega_{c}-\Gamma_{l}^{2} / 4 T_{e}\right)}{\Gamma_{l, l^{\prime}}} .
$$

In the opposite case $\left(\Delta_{l, l^{\prime}}<0\right)$,

$$
\begin{gathered}
\delta F_{l, l^{\prime}}\left(\omega_{c}\right)=\sum_{m=0}^{\infty} e^{-m \hbar \omega_{c} / T_{e}} I_{l, l^{\prime} ;-m}\left(\Delta_{l, l^{\prime}}\right) \times \\
\times \frac{\left(\Delta_{l, l^{\prime}}+m \hbar \omega_{c}-\Gamma_{l}^{2} / 4 T_{e}\right)}{\Gamma_{l, l^{\prime}}} .
\end{gathered}
$$

The corresponding form of $\delta H_{l, l^{\prime}}\left(\omega_{c}\right)$ differs from that of $\delta F_{l, l^{\prime}}\left(\omega_{c}\right)$ only by the additional factor $m$ inside the sum. One can see that $\delta F_{2,1}$ and $\delta F_{1,2}$ entering $\Phi\left(\omega_{c}\right)$ [as well as $\delta H_{2,1}$ and $\delta H_{1,2}$ in $\Theta\left(\omega_{c}\right)$ ] have opposite signs. This means that the contribution of scattering from $l=2$ to $l=1$ is not cancelled by the contribution of scattering from $l=1$ to $l=2$.

The conventional contribution to $\nu_{\text {eff }}$ originated from the expansion of $e^{\hbar \mathbf{q} \cdot \mathbf{v}_{\text {av }} / T_{e}}$ can be written as

$$
\begin{gathered}
\nu(B)=\frac{\nu_{0}^{(a)} \hbar^{2} \omega_{c}^{2}}{2 \pi^{1 / 2} T_{e}} \sum_{l, l^{\prime}} \frac{s_{l, l^{\prime}}}{\Gamma_{l, l^{\prime}}} n_{l^{\prime}} e^{-\Delta_{l, l^{\prime}} / T_{e}} \times \\
\times\left[\operatorname{coth}\left(\frac{\hbar \omega_{c}}{2 T_{e}}\right) F_{l, l^{\prime}}\left(\omega_{c}\right)+H_{l, l^{\prime}}\left(\omega_{c}\right)\right],
\end{gathered}
$$

where $F_{l, l^{\prime}}$ and $H_{l, l^{\prime}}$ are defined similar to $\delta F_{l, l^{\prime}}$ and $\delta H_{l, l^{\prime}}$ with the exception that their equations do not contain the factor $\left(\Delta_{l, l^{\prime}} \mp m \hbar \omega_{c}-\Gamma_{l}^{2} / 4 T_{e}\right) / \Gamma_{l, l^{\prime}}$. For SEs on liquid helium, Landau levels are extremely narrow: $\Gamma_{l} \ll T$. In this case, the collision frequency term of Eq. (5) originated from the expansion in $\hbar \mathbf{q} \cdot \mathbf{v}_{\mathrm{av}} / \Gamma_{l, l^{\prime}}$ acquires an additional large factor $T_{e} / \Gamma_{l, l^{\prime}} \gg 1$ as compared to the conventional term of Eq. ([6). Therefore,

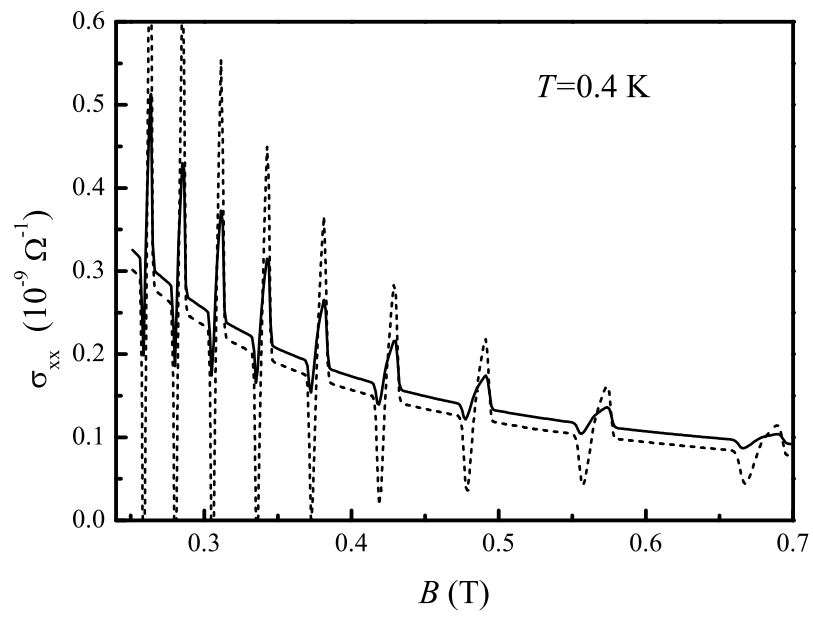

FIG. 1: Magnetoconductivity vs magnetic field for two MW power levels: $\Omega_{R} / \nu_{0}^{(a)}=0.2$ (solid) and $\Omega_{R} / \nu_{0}^{(a)}=0.4$ (dashed).

even a small nonequilibrium filling of the excited subband $n_{2}-n_{1} e^{-\Delta_{2,1} / T_{e}}>0$ can lead to giant oscillations in $\nu_{\text {eff. }}$.

The results of numerical evaluations of $\sigma_{x x}$, taking into account both the usual contribution to $\nu_{\text {eff }}$ defined by Eq. (6) and the sign-changing correction of Eq. (5), are shown in Fig. 1 for two levels of MW power defined by the ratio of the Rabi frequency $\Omega_{R}$ to $\nu_{0}^{(a)}$. Fractional occupancies $n_{l}$ are found from the rate equation of the two-subband model, while electron temperature $T_{e}$ is obtained from the energy balance equation. The MW resonance frequency equals $95 \mathrm{GHz}$. Other parameters correspond to experiments with liquid ${ }^{3} \mathrm{He}$ at $T=0.4 \mathrm{~K}$. One can see that for noninteracting electrons, the amplitude of the sign-changing correction to $\sigma_{x x}$ becomes larger in the low magnetic field range, and, at high MW powers, $\sigma_{x x}$ becomes negative. The shape of $\sigma_{x x}$ oscillations obtained here is in striking accordance with experimental data observed for SEs on liquid helium ${ }^{5}$. Conductivity minima occur when the parameter $\Delta_{2,1} / \hbar \omega_{c}$ is slightly above an integer, which also agrees with the observation.

The fractional occupancy of the second subband $n_{2}$ as a function of the parameter $\Delta_{2,1} / \hbar \omega_{c}$ is shown in Fig,2, It is reduced in the vicinity of the condition $\Delta_{2,1} / \hbar \omega_{c} \rightarrow$ $m$ (here $m$ is an integer) due to elastic inter-subband scattering.

The many-electron effect can be taken into account considering an electron moving in a quasi-uniform electric field of other electrons $E_{f}$ due to thermal fluctuations ${ }^{9}$. In this case, according to Ref. $\underline{6}, \Gamma_{l, l^{\prime}}$ entering $S_{l, l^{\prime}}^{(0)}(q, \omega)$ should be replaced by $\sqrt{\Gamma_{l, l^{\prime}}^{2}+x_{q} \Gamma_{C}^{2}}$, 


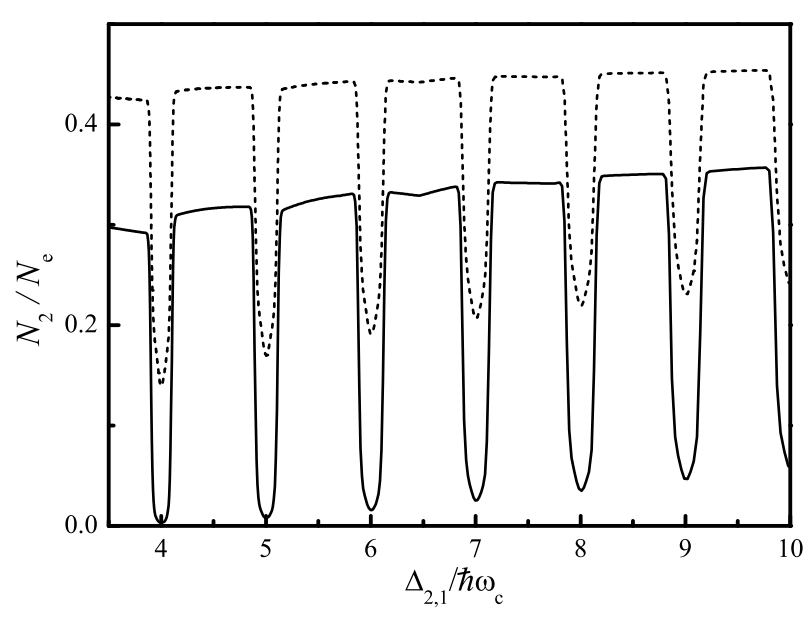

FIG. 2: The fractional occupancy of the second subband for two MW power levels: $\Omega_{R} / \nu_{0}^{(a)}=0.2$ (solid) and $\Omega_{R} / \nu_{0}^{(a)}=$ 0.4 (dashed).

where $\Gamma_{C}=\sqrt{2} e E_{f}^{(0)} l_{B}$, and $E_{f}^{(0)} \simeq 3 \sqrt{T_{e}} n_{s}^{3 / 4}$. Since $l_{B} \propto 1 / \sqrt{B}$, the many-electron effect broadens magnetooscillations and reduces their amplitudes in the low field range, which is also in accordance with experimental observations.

It should be noted that $\sigma_{x x}(B)$ curves do not change much even if we set $T_{e}=T$. Nevertheless, electron tem- perature as a function of the parameter $\Delta_{2,1} / \hbar \omega_{c}$ oscillates and rises when $\Delta_{2,1} / \hbar \omega_{c} \rightarrow m$. In this case, quasielastic decay transfers $\Delta_{2,1}$ to the kinetic energy of the inplane motion. At $\Omega_{R} / \nu_{0}^{(a)} \sim 0.2$, the maximum increase in $T_{e}$ is less than $2 \mathrm{~K}$ which allows to use the two-subband approximation. For high MW powers, under the condition $\Delta_{2,1} / \hbar \omega_{c} \rightarrow m$ electron population of higher subbands should be taken into account. Electron heating can be suppressed by increasing the holding electric field $E_{\perp}$ or by lowering the ambient temperature $T$. In the later case, electrons are predominately scattered by ripplons. Nevertheless, qualitatively magnetooscillations of $\sigma_{x x}$ should be the same because typical energy of ripplons involved is extremely small. There is also a possibility to use a higher MW resonance condition $\hbar \omega=\Delta_{l}-\Delta_{1}$ with $l>2$.

Concluding, we have shown that remarkable MWinduced oscillations of magnetoconductivity of SEs on liquid helium and zero-resistance states observed in Ref., 5 can be understood as a consequence of intersubband electron scattering by vapor atoms or ripplons under the condition that the fractional occupancy of the excited subband exceeds that given by the conventional Boltzmann factor. This explains why the MW resonance condition $\hbar \omega=\Delta_{2}-\Delta_{1}$ is crucial for observation of magnetooscillations and ZRS in this system. Electronelectron interaction, increasing with electron density, is shown to suppress the amplitude of magnetooscillations and to destroy the ZRS.

The author is grateful to K. Kono and D. Konstantinov for acquainting with experimental data before publication and for helpful discussions.
1 V. I. Ryzhii, Fiz. Tverd. Tela 11, 2577 (1969) [Sov. Phys. Solid State 11, 2078 (1970)].

2 R. Mani, J. H. Smet, K. von Klitzing,V. Narayanamurti, W. B. Johnson, and V. Umansky, Nature 420, 646 (2002).

3 A.V. Andreev, I.L. Aleiner, and A.J. Millis, Phys. Rev. Lett., 91, 056803 (2003).

4 D. Konstantinov and K. Kono, Phys. Rev. Lett. 103, 266808 (2009).

5 D. Konstantinov and K. Kono, arXive: condmat/1006.0349v2 (submitted to Phys. Rev. Lett.).
${ }^{6}$ Yu.P. Monarkha and K. Kono, Two-Dimensional Coulomb Liquids and Solids, Springer-Verlag, Berlin Heildelberg (2004).

7 Yu.P. Monarkha, D. Konstantinov, and K. Kono, J. Phys. Soc. Jpn. 76, 124702 (2007).

8 R.R. Gerhardts: Surf. Sci. 58, 227 (1976).

9 M.I.Dykman and L.S. Khazan, Zh. Eksp. Teor. Fiz. 771488 (1979) [Sov. Phys. JETP 50, 747 (1979)]. 